\title{
How to Achieve Cross-Cultural and Theoretical Diversities in Clinical Ethics Support Systems
}

\author{
Olukunle Cornelius Ewuoso* \\ Department of Philosophy, Stellenbosch University, South Africa
}

Received: 阱 August 25, 2018; Published: 眥 August 31, 2018

*Corresponding author: Cornelius Olukunle Ewuoso, Centre for Applied Ethics, Department of Philosophy, Stellenbosch University, Stellenbosch, Western Cape, South Africa

\section{Opinion}

There is a persistent call [1-3] for multiculturalism and diversity of theoretical and ethical frameworks in clinical ethics support systems (CESS) around the world. Undoubtedly, there is a lot a strategic think-tank or support system situated within the clinical context, to reflect on the various ethical issues health professionals face daily, can benefit from multiple ethical reasoning. This will optimize health care delivery and improve patient-care. In order to achieve multiculturalism or cross-cultural and theoretical diversities in CESS, there is a need for a respectful engagement between medicine and social sciences and the humanities. This engagement is mutually beneficial: the social sciences and the humanities can contribute a lot to medicine; similarly the humanities and social sciences can equally benefit from medicine. Medicine can share with the social sciences and humanities some of the ethical challenges it contends with daily, and the social sciences and humanities can contribute - to medicine - rigorous scientific methodologies and moral reasoning derived from different cultures and traditions.

In different studies [4-7], I have shown that the above engagement is not only desirable, but possible. In one study Ewuoso [4], I showed how a particular African ethics may be used to address the ethical impasse created by applying coercion in psychiatric care. Autonomous choices undeniably, are rights of patients. However, emphasizing rights for a mentally-ill patient could jeopardize the chances of the patient receiving care or endanger the public. Conversely, the beneficial effects of coercion are difficult to predict. Thus, applying coercion in psychiatric care requires delicate balancing of individual-rights, individual well-being and public safety, which previous models for applying coercion such as "Stone model" [8], "civil liberty approach" [9,10], "rehabilitation model" [11,12] and so on, were unable to achieve. These models mandate involuntary admission/hospitalization only when the individual constitutes a danger to the public/self; as well as when such involuntary admission and/or treatment will lead to recovery or rebirthing of autonomy. I argued that these models are problematic since they do not accommodate those who do not meet these conditions - that is, non-dangerous mentally ill individuals, whose conditions have no known treatment; but could benefit from other forms of treatment such as talk therapy. Hence, these models have limited relevance for care that is truly due to the mentally-ill.

To complement these models, I suggested a framework developed around the Yoruba concept of Omo-olu-iwabi, which grounds authentic personhood, as well as rightness of action in the extent to which it promotes harmonious relationships: ones in which individuals identify or care for each other's wellbeing and dignity. Within this framework, non-consensual treatment, continued hospitalization and/or physical restraint of individuals who are gravely disabled due to severe mental illness or chronic alcoholism; and in whom biological treatment has failed, are not necessarily tyrannical, but are justified since such coercive measures promote individual health by requiring the community to actively work for the well-being of all. Furthermore, this coercive measure facilitates public good by ensuring that those who pose significant risk to others are involuntarily constrained. It is in this regard that the ethical approach built around the Yoruba concept of Omo-olu-iwabi balances the three competing interests: individual rights, health/safety of mentally ill and public good. In another study [7], I argued the thesis that a set of guidelines, firmly rooted in a particular interpretation of African moral theory, specifically, Ubuntu - will do a better job than current medical ethics frameworks, in addressing ethical challenges around misattributed parentage within the clinical context.

Incidental information such as information with significant personal/health implications raises unique challenges for medical professionals. For example, withholding information of misattributed paternity accidentally discovered in clinical interactions may be seen by a patient as a violation of his/her rightto-know. Contrarily, disclosure where a patient has not requested information - or where establishing paternity is not the purpose of clinical visit/interaction - may be taken by the patient as a violation of his/her right 'not-to-know'. Resolving these challenges remains a herculean task. African moral theory contains an under- 
emphasized value for addressing such ethical challenges around misattributed parentage in the field of transplant. In that study, I contributed that knowledge in ways that are likely to enhance clinician-patient relationship. I encourage similar engagements - which focus on how various ethical frameworks may be used to address specific dilemmas within the clinical context - with medicine. Future studies, for example, can address other dilemmas by appealing to other ethical frameworks such as Islamic ethics, Asian ethics and so on. I strongly believe that these discussions and engagements between medicine and the humanities and social sciences are necessary for achieving cross-cultural and theoretical diversities in CESS.

As different scholars attempt to engage medicine and ethical issues within the clinical setting, from a variety of backgrounds within the social sciences and humanities, some pitfalls must be avoided: when intellectuals introduce alternative theoretical or ethical frameworks, very often critics measure the new framework by appealing to existing norm. This is problematic, since existing norm has dominance not only on the way health professionals address real ethical issues, but also the way they evaluate new ideas. This can prevent authentic reading of what the new framework suggests. Each newly introduced framework should be considered within the purview of an alternative realism: as a valuable and plausible approach for thinking around ethical issues within the clinical context. I conclude by emphasizing that achieving crosscultural and theoretical diversities in CESS is an imperative: the mere fact that patients, doctors, systems and communities have varying value systems, reveal that no "single" norm should have complete monopoly in CESS. I recommend that more studies should give exposure to lesser known theoretical and ethical frameworks from other regions of the world. And I am optimistic that if these well-defended and articulated alternative frameworks are incorporated into current medical codes and curricula, they will significantly enhance health care delivery and health professionalpatient relationship; as well as bring us closer to accomplishing cross-cultural and theoretical diversities in clinical ethics support systems.

\section{References}

1. Westra AE, Willems DL, Smit BJ (2009) Communicating with Muslim parents: "the four principles" are not as culturally neutral as suggested. Eur J Pediatr 168(11): 1383-1387.

2. Genuis SJ, Lipp C (2013) Ethical diversity and the role of conscience in clinical medicine. Int J Family Med 2013: 587541.

3. Jegede AS (2009) African Ethics, Health Care Research and Community and Individual Participation. Journal of Asian and African Studies 44(2): 239-256.

4. Ewuoso CO (2016) Beneficial Coercion in Psychiatric Care: Insights from African Ethico-Cultural System. Dev World Bioeth 18(2): 91-97.

5. Ewuoso C (2017) Models for truth-telling in physician-patient encounters: what can we learn from Yoruba concept of Ooto? Dev World Bioeth.

6. Ewuoso OC, Hall S, Dierickx K (2017) How do healthcare professionals manage ethical challenges regarding information in health care professional-patient clinical interactions? A Review of Concept/ Argument-Based Papers and Case Analyses. South African Journal of Bioethics and Law 10(2): 75-82.

7. Ewuoso C (2018) Managing ethical challenges around misattributed parentage within the clinical context: Insights from an African moral theory. Dev World Bioeth.

8. Stone A (1975) Mental Health and Law: A System in Transition, Rockville, Maryland, National Institute of Mental Health. Psychiatric Services 27(7): 525-525.

9. Zhang S, Mellsop G, Brink J, Wang X (2015) Involuntary admission and treatment of patients with mental disorder. Neuroscience Bulletin 31(1): 99-112.

10. Steinert T, Noorthoorn EO, Mulder CL (2014) The Use of Coercive Interventions in Mental Health Care in Germany and the Netherlands. A Comparison of the Developments in Two Neighboring Countries. Frontiers in Public Health 2: 141.

11. Caplan A (2008) Denying autonomy in order to create it: the paradox of forcing treatment upon addicts. Addiction 103(12): 1919-1921.

12. Caplan AL (2006) Ethical issues surrounding forced, mandated, or coerced treatment. J Subst Abuse Treat 31: 117-120.
ISSN: 2574-1241

DOI: 10.26717/BJSTR.2018.08.001673

Cornelius Olukunle Ewuoso. Biomed J Sci \& Tech Res

This work is licensed under Creative Commons Attribution 4.0 License

Submission Link: https://biomedres.us/submit-manuscript.php

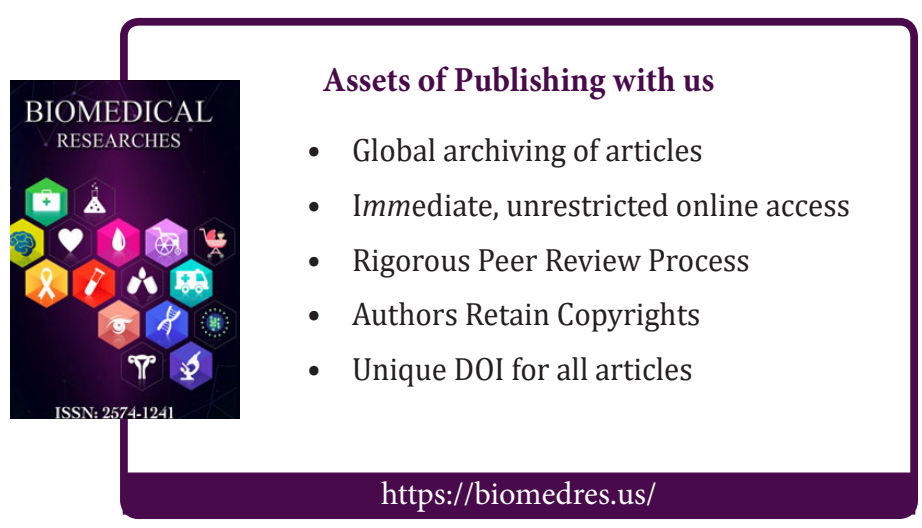

